\title{
Experimental evidence of a fractal dissipative regime in high- $\mathrm{T}_{c}$ superconductors
}

\author{
Mladen Prester \\ Institute of Physics, P.O.B. 304, HR-10 000 Zagreb, Croatia
}

(May 18, 2018)

\begin{abstract}
We report on our experimental evidence of a substantial geometrical ingredient characterizing the problem of incipient dissipation in high- $\mathrm{T}_{c}$ superconductors (HTS): high-resolution studies of differential resistance-current characteristics in absence of magnetic field enabled us to identify and quantify the fractal dissipative regime inside which the actual current-carrying medium is an object of fractal geometry. The discovery of a fractal regime proves the reality and consistency of criticalphenomena scenario as a model for dissipation in inhomogeneous and disordered HTS, gives the experimentally-based value of the relevant finite-size scaling exponent and offers some interesting new guidelines to the problem of pairing mechanisms in HTS.
\end{abstract}

74.50.-g, 64.60.Ak, 74.50.+r

Local inhomogeneities characterize HTS both on nanoscopic [1,2] (e.g., periodic or aperiodic variation of local oxygen stoichiometry) and mesoscopic [3] (e.g., oxygen depleted grain boundaries) spatial scales. While all the consequences on normal and superconducting charge transport in the former case has not been entirely clarified yet the case of grain boundaries is better understood: at least in a broad range of experimental parameters the supercurrent transport in polycrystalline samples relies on 'weak link network' (WLN), i.e., on mesoscopic superconducting islands interconnected by Josephson interaction. Although the transport features on nanoscopic scale may significantly differ from those characterizing a rather simple WLN problem (e.g., local inhomogeneities seem to give rise, as reviewed by Refs. [1,2, to conducting stripes, clusters, wires or filaments which are, at least to some extent, mobile, compared to predominantly static weak links) the experimental evidence in favor of $a$ - $b$ plane Josephson junctions [5] indicates that, besides qualitative similarities, the intrinsic and WLN transport are more closely related one to another than it had been foreseen earlier.

Irrespective of the extent the processes at nano- and mesoscopic scales are related, the problem of charge transport in WLN represents an autonomous subject of much interest due to its relevance for general understanding of transport in heterogeneous systems and in Josephson Junction Arrays (JJA) in particular [6]. Focusing to the problem of dissipation there are convincing arguments, particularly in absence (or in small) magnetic fields, that the onset of dissipation is dominated rather by a phenomenon of percolation than the dynamical features of flux lattice [7, 4]. In a disordered-bonds (DB) model [4, 3] the critical current $I_{c}$ characterizing the dissipation onset reflects the connectivity threshold $p_{c}$ of classical percolation networks 9,10 (such that $p_{c}=p\left(I_{c}\right)$ ) so that the experimentally documented power-law-like currentvoltage (I-V) characteristics can be naturally interpreted as a current-induced but in essence traditional critical phenomenon. Consequently, I-V characteristics should also reveal various manifestations of crossover between the relevant length scales known to underly the critical behavior. We show in this report that the latter crossover may be detected and quantitatively investigated in experimental I-V curves. In particular, we claim that the I-V characteristics are generically composed of the three distinct regimes: a regime revealing no practical dissipation, a regime obeying conventional correlation length scaling (homogeneous regime), and an intermediate regime obeying finite-size scaling (fractal regime). While the dissipation in the former regimes has been already a subject to experimental reports and appropriate modeling [4, \&], the experimental results concerning the fractal regime are reported here for the first time.

The basis of the model is the idea that the increasing current applied to disordered WLN decreases the fraction of Josephson-current-carrying bonds in a random manner. Hence, the applied current plays the role of random generator which in classical random electrical networks changes the relative fractions of their components. In DB model [4,3] the elements and the relevance of this analogy has been studied in details. Here we focus to the problem of relevant length scales. In analogous classical networks there are two of them [9, 10]: the correlation length $\xi$ (the representative size of growing ramified clusters ) for $p$ away from $p_{c}$ and the sample size $L$ for $p$ close to $p_{c}$ ("at criticality"). In approaching $p_{c}, \xi$ diverges involving exponent $\nu(\approx 0.88$ in $3 \mathrm{D}$ systems $)$ and the power-law form of static and dynamic quantities manifests the property of spatial scaling. In particular, the resistance $R$ of the random-superconductor network (RSN) disappears 10 as $\left(p_{c}-p\right)^{s} \propto \xi^{-s / \nu}$, where $s$ is the breakdown exponent $(s \approx 0.8$ in $3 \mathrm{D})$. Close to $p_{c}$ the homogeneous-tofractal transformation of the geometry of incipient cluster takes place and, while $R$ becomes independent of $p$, the finite-size scaling relation [10,9] $R(L) \propto L^{-s / \nu-1}$ replaces the ordinary $R(L) \propto L^{-1}$ one. In applying a similar scenario to current-induced transition in WLN we 


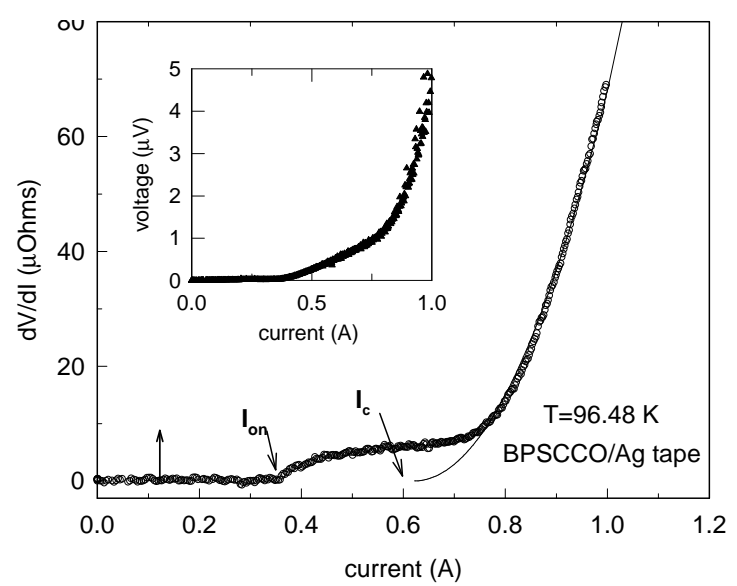

FIG. 1. High-resolution differential resistance in Ag/HTS composite tape, core thickness $\approx 30 \mu \mathrm{m}$. The pronounced anomaly above the sharp dissipation onset at $I_{\text {on }}$ is attributed to the sample size scaling $(\xi \geq L)$. For higher currents the usual correlation length scaling $\left(\propto \xi^{-x / \nu}\right)$ takes over. Experimentally, $d V / d I \propto\left(I-I_{c}\right)^{x}, I_{c}=0.6, x=2$, as shown by the thin line. Vertical arrow illustrates the resolution: Its length corresponds to $d c$ voltage of $15 n V$. The inset: $V-I$ curve obtained by numerical integration of the measured $d V / d I-I$.

assume that, in approaching $I_{c}$ (i.e., $p_{c}$ ) from above, the representative size of the largest phase coherent cluster diverges as $\xi$ as well. The experimental studies [ 4 , of the related homogeneous regime were shown to be in a close agreement with the predictions of the model. However, the precise interpretation [11] of the characteristic exponent (experimentally, $d V / d I$ exponent is close to 2 ) is still unresolved (see discussion section). Considering the experimental accessibility of the crossover to the fractal regime we note that the unit of length involved in the WLN problem (i.e., average grain size $\ell$ ) belongs to mesoscopic $(\mu \mathrm{m})$ scale. Hence, the observation of a size effect introduced by competing length scales seems, for polycrystalline samples of reduced but still macroscopic thickness, as an open possibility. Indeed, the first high resolution dynamical resistance measurements 12 (achieving the equivalent voltage resolution of better than $1 \mathrm{nV}$ ) on polycrystalline samples which are thin by their very design, HTS superconductor/normal metal composite tapes (superconducting core thickness in the range 10-50 $\mu \mathrm{m}$ ), revealed the two characteristic currents. As illustrated by Fig. 1, the lower one triggers the onset of low-level, non-exponential dissipation (onset current, $I_{o n}$ ) while the higher one parameterizes the scaling behavior, $d V / d I \propto\left(I-I_{c}\right)^{x}, x \approx 2$, of subsequently rapidly growing dissipation (thermodynamical critical current, $I_{c}$ ). One could assume that a rather broad dissipative range between $I_{o n}$ and $I_{c}$ corresponds to validity of $\xi \geq L$ when the incipient dissipative sites would fill the sample-sized network of fractal geometry. Indeed, the saturation-like behavior of dissipation in that range is, while in obvious disagreement with any fluxcreep model (exponential in applied current), at least in qualitative agreement with general independence of any observable $(d V / d I$ in our case) on $\xi$ in the range of sample-sized fractal [9, 10]. A similar observation of the broad range of low-level dissipation in composite tapes has also been reported by other authors but interpreted by less fundamental causes [13].

By studying a thickness dependence in appropriate samples we prove now the presence of geometrical constraints of fractal nature in initial dissipation of HTS in a more quantitative way (the results on composite tapes, Fig. I, represent just a qualitative indication). The presence of many spurious and/or overlapping effects [14] in composite tapes and HTS films precludes obtaining a firm quantitative information on critical behavior from these samples. We performed therefore the measurements of $\mathrm{I}-\mathrm{dV} / \mathrm{dI}$ characteristics on a wellcharacterized, non-textured (i.e., isotropic) polycrystalline $\mathrm{RBa}_{2} \mathrm{Cu}_{3} \mathrm{O}_{7-x}(\mathrm{R}=\mathrm{Y}, \mathrm{Gd}$ ) bulk sample (a WLN prototype!) in many successive steps, after its thickness had been gradually reduced by fine plan-parallel grinding. In that way, apart from various thickness, all the measurements were performed on the same initial sample. The transport properties of the sample (e.g., room temperature resistivity, $\mathrm{T}_{c}$, resistive transition width) did not change in all stages of its thickness. The measurements we report on in this paper covered the sample thickness range of 20-1000 $\mu \mathrm{m}$ (factor of 50). For thicknesses above approximately $60 \mu \mathrm{m}$ only the unique 'thermodynamic' critical current, accompanied by the usual power-law-like $\left(\propto\left(I-I_{c}\right)^{x}, x \approx 2\right)$ growth of dissipation (specific for scaling regime in a very large sample, $\xi \ll L$ ), have been detected. In the sample stages involving all smaller thicknesses the two characteristic currents, $I_{o n}$ and $I_{c}$, have been observed, just as in composite tapes. Some of the experimental $d V / d I(L)$ curves were shown in Fig. 2 using moderate (main figure) and a very high dynamical resistance resolution (inset). The anomalous dissipative range between $I_{o n}$ and $I_{c}$ is rather complex but systematically depends on sample thickness: The size of the onset anomaly drastically increases by decreasing $L$. The analysis and interpretation have been performed inside the DB model [3, 3 ] which provides both the limiting behavior in the correlation length scaling range $\xi \ll L$ (thin lines in Figs. 1, 2) and the estimate of the width of the range of sample size scaling $(\xi \geq L)$. The crossover between these two ranges takes place when the diverging $\xi, \xi(p)=\ell\left|p-p_{c}\right|^{-\nu}$, becomes equal or higher than the sample size. The unit of length is $\ell$, the network unit cell size (for $\ell$ we used $\ell \approx 5 \mu m$, the average grain size of the sample). In other words, as long as the fraction of 'good' bonds deviates from $p_{c}$ (percolation threshold of an infinite system) by $\Delta p=(\ell / L)^{1 / \nu}=(\ell / L)^{1.136}$ or less, the 


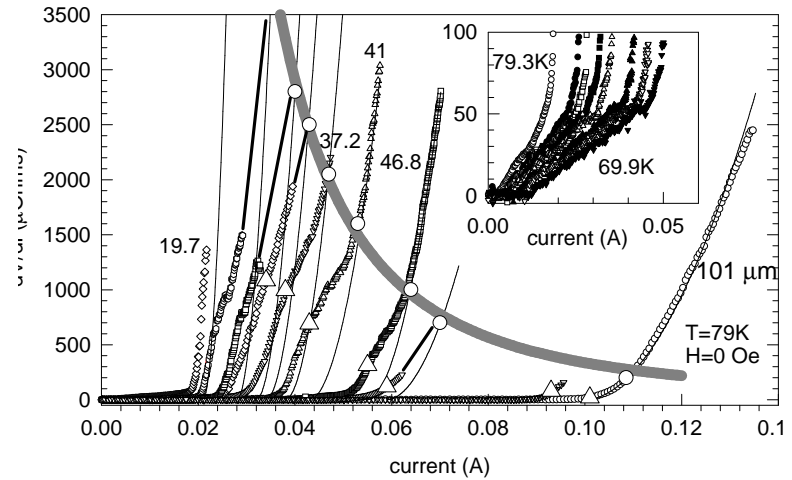

FIG. 2. Thickness dependence of differential resistances of polycrystalline $\mathrm{GdBa}_{2} \mathrm{Cu}_{3} \mathrm{O}_{7-x}$ sample (open symbols) and homogeneous-to-fractal phase boundary (thick grey line). The thickness (in $\mu m$ ) is designated by numbers near the experimental curves. The thin lines represent the predicted power-laws characterizing the homogeneous regime (see text). A sizable deviation which scale the sample size corresponds to sample-sized cluster of fractal geometry. Large triangles were used to extract the $R_{c}(L)$ scaling in Inset to Fig.3. Inset: Temperature dependence of the very onset of anomalous dissipation in the thinnest $(19.7 \mu \mathrm{m})$ sample.

macroscopic properties should have a weak dependence on $p$ (i.e., on current in our case) and the underlying ramified sample-sized cluster should be, geometrically, a fractal.

In DB model 44,3] a linear $p(I)$ approximation has been shown to work well close to $p_{c}$ (but still outside $\Delta p): p_{c}-p=\left(c_{2} / c_{1}\right)\left(p_{c} / I_{c}\right)\left(I-I_{c}\right)$ where $c_{2} / c_{1}(\equiv g$ henceforth) is a geometrical factor of order 1 . The current interval compatible with $\Delta p, \Delta I$, reads therefore $\Delta I=I_{c} g\left(\Delta p / p_{c}\right)=\left(I_{c} g / p_{c}\right)(\ell / L)^{1.136}$. The onset current can be now defined as $I_{o n}=I_{c}-\Delta I$ and the corresponding current density is expected to depend strongly on sample thickness $L: J_{\text {on }}=J_{c}\left(1-\left(g / p_{c}\right)(\ell / L)^{1.136}\right)$. Experimentally, while the determination of $I_{o n}$ in thin samples is quite straightforward (the onset of dynamical resistance is very sharp, inset to Fig. 2) the determination of $I_{c}$ is not; $I_{c}$ represent just a parameter in DB model for dissipation [4], $d V / d I=R_{f}\left(g p_{c} / I_{c}\right)^{x}\left(I-I_{c}\right)^{x}$, where $R_{f}$ represents the total resistance of WLN in homogeneous regime.

An interesting observation is that the thermodynamic critical current density $J_{c}$ is strongly thickness dependent as well, Fig. 3. The presence of a size-effect in $J_{c}$ is, however, a rather well-known [15] although somewhat neglected phenomenon. We found that the observed increase of $J_{c}$ for a factor of 5 by thinning the cross-section fits perfectly the general breakdown formula [16], $J_{c} \propto 1 /\left(1+\left(a_{\log _{10}} L\right)^{\alpha}\right)$ which predicts that the critical current density vanishes in thermodynamic limit.

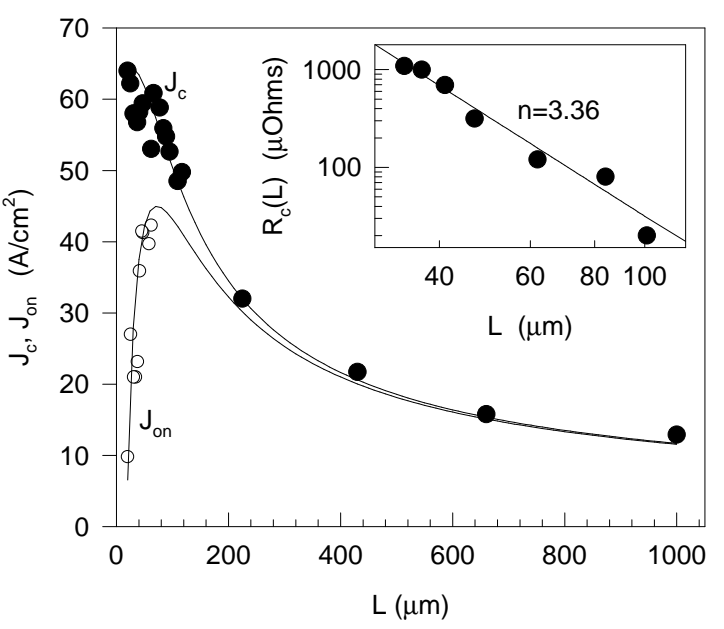

FIG. 3. Thickness dependence of thermodynamical critical current $\left(J_{c}\right)$ and onset current $\left(J_{o n}\right)$ density- experimental points (symbols) and model predications (thin lines). Inset: Thickness dependence of $d V / d I$ at $I_{c}$ (at criticality) indicates the fractal geometry of the network. The slope for homogeneous (Euclidean) electrical networks is $n=1$.

Our results are compatible with $J_{c}(L)=5 J_{c}\left(L_{\max }\right) /(1+$ $\left.\left(\log _{10}\left(L / L_{\min }\right)\right)^{3}\right)$ where $L_{\max }$ and $L_{\min }$ are maximal and minimal sample thickness, respectively. Inserting the latter expression into the derived one for $J_{o n}$ (comprising only one adjusting parameter, $g$ ) one gets the peaked curve, Fig. 3, as a prediction of this model. The two dependencies (for $J_{c}$ and $J_{o n}$ ) joins smoothly in increasing $L$ as $\Delta p$ (i.e., $\Delta I)$ continuously vanishes, as well as $\xi$ itself, in this limit. The remarkable overlap of experimental points and the model predictions, Figs.2,3, illustrate the reality of the model, in spite of its simplicity. It is interesting to note that Fig. 2 can be interpreted as a kind of phase diagram: the thick grey line separates the homogeneous from the fractal phase of the cluster inside which the initial dissipation grows.

The main quantitative results of this work is plotted as an Inset to Fig. 3. It plots the value of dynamical resistance in fractal phase $R_{c}\left(I_{c}\right)$ for each available sample thickness $L$. The particular current at which $R_{c}$ has been taken was $I_{c}$, the representative of $p_{c}$ in currentinduced transitions. The $R_{c}(L)$ relationship can be fit nicely by a power-law $R_{c}(L) \propto L^{-n}$. The exponent value $(n=3.36)$ deviates strongly from the value $n=1$ characterizing homogeneous networks (the scaling with $n=1$ is strictly obeyed in, e.g., $R_{f}(L)$ dependence). Also, the quantity $\nu(n-1)$ which in finite-size scaling calculations gives the dynamical exponent of homogeneous regime is numerically very close $(=2.1)$ to experimentally welldocumented [4] value $x=2$ valid in that regime. Both arguments provide therefore the evidence for fractal geometry involved in initial dissipation. 
The important issue which remains to be clarified is the interpretation of the value of the exponent $n$, as well as of the related exponent $x$ characterizing the homogeneous regime of I-V characteristics. The experimental values of exponents $(x \approx 2, n \approx 3.4)$ are, while mutually consistent, in clear disagreement with those obtained by identifying RSN and WLN, i.e., $x \approx s \approx 0.8, n=s / \nu+1 \approx 1.9$. There could be several reasons why the WLN exponent, $x$, may differ from the classical one, $s$. A well-known example is the 'Swiss-cheese' morphology in continuum percolation [18], equivalent to the case of broad distribution in bond resistances, shown to influence the exponent. The other is experimentally documented nonuniversal conduction in carbon-black-polymer composite [19] attributed to peculiarities of tunneling as a mechanism of local conduction. Both the broad distribution and tunneling seems as natural possible causes for exponent deviation in WLN of HTS. We also note that there are some obvious differences between current-generated (WLN) and random-generated (RSN) clusters. Better understanding of these differences could probably come from very recent and exciting studies of self-organized [20] and 'small-world' 21] networks.

The observation of a fractal dissipative regime offers an interesting new guideline towards understanding the intrinsic pairing interaction in HTS. There are namely numerous arguments that the intrinsic intra- and interplane charge transport takes place actually in a heterogeneous conductive medium [1], with percolation playing probably the important role as well [2]. Moreover, these conditions are considered, according to some authors [22], as substantial ingredients of the mechanism of superconductivity itself. The involved heterogeneity may rely either on charge separation, stripes, wires, etc., [1], cluster formation [2] or on filamentary fragmentation [22]. Under these circumstances it seems quite reasonable to assume that the intrinsic current transfer may include the fractal network as well (at least in certain range of relevant transport parameters). Given the electrically heterogeneous local properties, combined with vicinity of metal-insulator transition, the associated elastical (vibrational) network (which is formally isomorphic to its electrical counterpart 10]) might be not only heterogeneous but may posses a fractal geometry as well. In a fractal elastical lattice the vibrations are, instead of extended phonons, the localized high-frequency fractons 23,24 which may contribute in pairing. Relaying on peculiarities of the fracton density of state [23], such as high cut-off frequency and/or high-frequency 'missing modes' [23], the pairing temperatures could be higher than those associated to classical phonons. On basis of our results we suggest therefore consideration of a fractal dynamical lattice as a possible source of non-standard pairing interactions at high temperatures.

The author acknowledges fruitful exchange of ideas with J.C.Phillips and is also indebted to K.Uzelac and
I. Živković for numerous discussions and to D.Pavuna for his continuous interest and support. I am also grateful to P.Kováč and F.C.Matacotta for providing me with samples.

[1] D.Mihailovic and K.A.Müller in High-T $T_{c}$ superconductivity 1996: Ten years after the discovery E.Kaldis, Editor, Kluwer Academic Publisher, 243 (1997), and references therein.

[2] J.Mesot and A. Furrer, Journal of Superconductivity 10, 623 (1997), and references therein.

[3] M.Prester, Supercond. Sci.Technol.11, 333 (1998), and references therein.

[4] M.Prester, Phys.Rev.B54, 606 (1996).

[5] H.Darhmaoui and J.Jung, Phys.Rev.B 57, 8009 (1998).

[6] See, e.g., Coherence in Superconducting Networks, edited by J.E.Mooij and G.B.J Schőn, Physica (Amsterdam) B152 (1988).

[7] A.E.Pashitskii. et al., Science 275, 367 (1997).

[8] E.Granato and D.Dominquez, Phys.Rev.B 5614671 (1997).

[9] D.Stauffer and A.Aharony, Introduction to Percolation Theory (Taylor and Francis, London, 1992)

[10] Fractals and Disordered Systems, edited by A.Bunde and S.Havlin (Springer-Verlag, Berlin, 1991).

[11] The closeness of $x$ to the value of conductivity exponent $(t \approx 2)$ inspired some speculations (Ref. 44) about actual identity of these exponents. We are however unaware of a convincing general argument equating $x$ with $t$ at present.

[12] M.Prester, P.Kováč, and I.Hušek in Oxide superconductors and Nano-Engineering III, edited by I.Bozovic and D.Pavuna, Proc.SPIE 3481 (1998), in press.

[13] M.Polak et al., Supercond.Sci.Technol. 10, 769 (1997).

[14] E.g., in composite tapes -redistribution of (super)currents between the core and the normal metal, the presence of the superior-quality layer at their interface, in thin films - a complicated dimensional cross over, Berezinskii-Kosterliz-Thouless scenario and specific dynamics of thermally excited vortex-antivortex pairs in thin films, anisotropy effects in both systems, etc.

[15] H.Dersch and G.Blatter, Phys.Rev.B 36, 11391 (1988); E.Babić et al. Phys.Rev.B 43913 (1992).

[16] M.Duxbury, P.D.Beale and P.L.Leath, Phys.Rev.Lett. 57, 1052(1986); P.L.Leath and W.Tang, Phys.Rev.B 39, 6485 (1989), and references therein.

[17] M.N.Barber in Phase Transition and Critical Phenomena, Volume 8, edited by C.Domb and J.L.Lebowitz (Academic Press, London 1983), and the references therein.

[18] B.I.Halperin, S.Feng, and P.N.Sen, Phys.Rev.Lett.54, 2391 (1985)

[19] I.Balberg, Phys.Rev.Lett.59, 1305 (1987).

[20] K.Christensen et al. Phys.Rev.Lett.81, 2380 (1998).

[21] D.J.Watts and S.H.Strogatz, Nature 393, 440 (1998).

[22] J.C.Phillips, Physica C 252, 188 (1995); J.C.Phillips, in 
Superconducting and Related Oxides: Physics and $\mathrm{Na}$ noengineering III, edited by D.Pavuna and I.Bozovic, Proc.SPIE 3481 (1998), in press.

[23] R.Orbach, Science 231, 814(1986); T.Nakayama, K.Yakubo, and R.Orbach, Rev.Mod.Phys. 66, 381 (1994).

[24] For a relevant recent report on the crossover between phonon and fracton modes in complex conductance studies of JJA see A.-L.Eichenberg et.al, Phys.Rev.Lett.77, 3905 (1996). 